health were associated with physical fatigue, but only higher muscular strength is associate with lower mental fatigue score $(\mathrm{p}=0.038)$. In multivariate analysis, muscular strength and balance were independently associated with fatigued, OR of 0.94 (95\% CI: 0.90 to 0.99 ) and 0.95 (95\% CI: 0.92 to 0.99 ) respectively.

Conclusion Physical health and fatigue are related. Impact of physical health interventions on fatigue would require further studies.

\section{COMPARING DISABILITY AND RETURN TO WORK OUTCOMES BETWEEN ALTERNATIVE AND TRADITIONAL WORKERS' COMPENSATION PROGRAMS}

${ }^{1}$ A Ryan*, ${ }^{2} \mathrm{~K}$ Schofield. 'University of Minnesota Minneapolis, USA; ${ }^{2}$ University of Minnesota Duluth, USA

\subsection{6/oemed-2018-ICOHabstracts. 1579}

Introduction In 1995, Minnesota, United States, passed legislation to allow for an alternative workers' compensation system and the Union Construction Workers' Compensation Program (UCWCP) was developed. Its goals include the use of preapproved medical and rehabilitation providers, dispute resolution, and prioritising a quick and safe return to work. The aim of this study is to determine if differences in recoveryrelated outcomes exist between UCWCP and traditional workers' compensation programs (TWCP).

Methods Workers' compensation claim data for the period 2003-2016 were obtained from the Minnesota Department of Labour and Industry. Claims were classified as processed through UCWCP or TWCP. Outcomes for this study included: permanent partial disability (PPD) and Temporary Total Disability (TTD). The relative risk (RR) of incurring PPD and sustained TTD in UCWCP versus TWCP was calculated using $\log$ binomial regression. Ordered logistic regression models were utilised to calculate the odds of higher percentages of PPD in UCWCP. All estimates were adjusted for age, gender, and insurance carrier.

Results Of 33682 claims, 3269 (9.7\%) were processed through UCWCP. The mean claim durations for UCWCP and TWCP were 54 and 49 weeks, respectively. While UCWCP claims, versus TWCP, were associated with an increased risk of PPD $(R R=1.2,95 \% \mathrm{CI}$ : 1.1 to 1.3$)$, they had increased odds of lower percent PPD (Odds Ratio=1.2, 95\% CI: 1.1 to 1.4). Sustained TTD was more likely in UCWCP compared to TWCP (RR $=2.0,95 \% \mathrm{CI}: 1.4$ to 3.0$)$.

Conclusions While the increased risks of permanent partial and sustained temporary total disability ratings among UCWCP claims may reflect higher severity, the lower percentage of disability could result from improvements in access and quality of healthcare treatments. Further work will identify and control for relevant severity indicators.

\section{SWIMMING AGAINST THE CURRENT: A META- ETHNOGRAPHY EXAMINING LIVING WITH ARTHRITIS AND BEING EMPLOYED}

RJ Purc-Stephenson*, H Smith, J Dostie. University of Alberta, Camrose, Canada
Introduction Arthritis and related rheumatic conditions are common causes of work disability in Canada and the United States, with job loss ranging between 37\%-60\% within the first 10 years of diagnosis. As diagnosis commonly occurs between the ages of 30-60 years, the symptoms of pain, limited mobility, and fatigue can seriously disrupt and hinder work lives. While research has examined disability and work loss among persons with arthritis (PwA), little is known about what they do to maintain employment. Our goal was to understand how PwA experience employment and to use this information to build a model describing what they need to sustain employment.

Methods We searched published studies on arthritis and employment from six electronic databases (1980-2017) and bibliographical reviews using a combination of keywords related to arthritis, employment, and qualitative research. Our search yielded 748 articles, and after applying the inclusion criteria, 17 studies remained. Two reviewers independently reviewed, critically appraised, and extracted concepts from each study in chronological order.

Result Using a meta-ethnographic process, we identified seven themes highlighting the common issues experienced by PwA. Using these themes, we developed a process model that illustrates how individual factors (i.e., physical symptoms, selfawareness, meaning of work) influence work-sustainability strategies that are initially privately managed (i.e., personal adjustments, medical treatment, family support); however, when symptoms become too difficult to conceal, individuals will disclose their condition to their employer so that they can draw upon additional work-sustainability strategies (i.e., work accommodations, supervisor and co-worker support, insurers). Individuals engage in these strategies to maintain a 'non-ill' identity and remain in their current job for as long as possible.

Discussion Our findings will help rehabilitation specialists, employers, and researchers understand what PwA may need to sustain meaningful employment outcomes. Implications to workplace policies and practices are discussed.

\section{A TAILORED WORK- RELATED SUPPORT INTERVENTION FOR GASTRO-INTESTINAL CANCER PATIENTS: INTERVENTION PROTOCOL}

${ }^{1}$ AnneClaire GNM Zaman*, 'Angela GEM de Boer, ${ }^{2}$ Kristien MAJ Tytgat, ${ }^{3,4}$ Jean HG Klinkenbijl, 'Monique HW Frings-Dresen. 'Academic Medical Centre, University of Amsterdam, Department Coronel Institute of Occupational Health, Amsterdam Public Health research institute, Amsterdam The Netherlands; ${ }^{2}$ Academic Medical Centre, University of Amsterdam, Department of gastroenterology, Amsterdam, The Netherlands; ${ }^{3}$ Gelre Hospital, Department of Surgery, Apeldoorn, The Netherlands; ${ }^{4}$ University of Amsterdam, Amsterdam, The Netherlands

\subsection{6/oemed-2018-ICOHabstracts. 1581}

Introduction The objective was to develop a tailored intervention for work- related problems to support gastro-intestinal (GI) cancer patients and to evaluate its cost-effectiveness compared with the usual care provided.

Methods We designed a multicentre randomised controlled trial with a follow-up time of twelve months. Included participants will be aged 18-63 years, diagnosed with a curative primary GI cancer and employed at time of diagnosis. The participants will be randomised to the intervention or to usual care. 